\title{
COMPARATIVE PRECISION OF THE PIVOTAL ESTIMATORS OF PARTICLE SIZE
}

\author{
LUIS M CRUZ-ORIVE \\ Department of Mathematics, Statistics and Computation, Faculty of Sciences, University of Cantabria, Avda. \\ Los Castros s/n, E-39005 Santander, Spain \\ (Accepted January 14, 2008)
}

\begin{abstract}
The pivotal estimators of the surface area and the volume of a generic "particle" are based on a point sampled test line on an isotropic pivotal plane through a fixed point called the pivotal point. The purpose of this paper is to compare the precision of the pivotal estimator of surface area against the surfactor on the one hand, and of the pivotal estimator of volume against the nucleator on the other. For the sake of a tractable but informative model we compute exact variances for a spherical particle with an eccentric nucleolus.
\end{abstract}

Keywords: convex body, invariant test line, particle, pivotal point, pivotal section, point sampled test line, stereology, support function, support set, surface area, volume.

\section{INTRODUCTION}

A new representation has been described for the invariant density of a straight line $L_{1}^{3}$ in $\mathbb{R}^{3}$ (Varga, 1935; Cruz-Orive, 2005). With reference to Fig. 1, consider a fixed point $O$, called the pivotal point, an isotropic plane $L_{2[0]}^{3}$ through $O$, called the pivotal plane, and a point $z$ endowed with the uniform density on $L_{2[0]}^{3}$. The classical construction establishes that a straight line $L_{1}^{3}$ through $z$ and normal to the pivotal plane is invariant with respect to motions. The new representation implies that the density of a straight line $L_{1[\cdot]}^{2}$ normal to the axis $O z$ at the point $z$, and contained in the pivotal plane, is also invariant with respect to motions in $\mathbb{R}^{3}$. In other words, a point sampled test line $L_{1[\cdot]}^{2}$ in a pivotal plane has exactly the same properties as an invariant test line $L_{1}^{3}$ in $\mathbb{R}^{3}$.

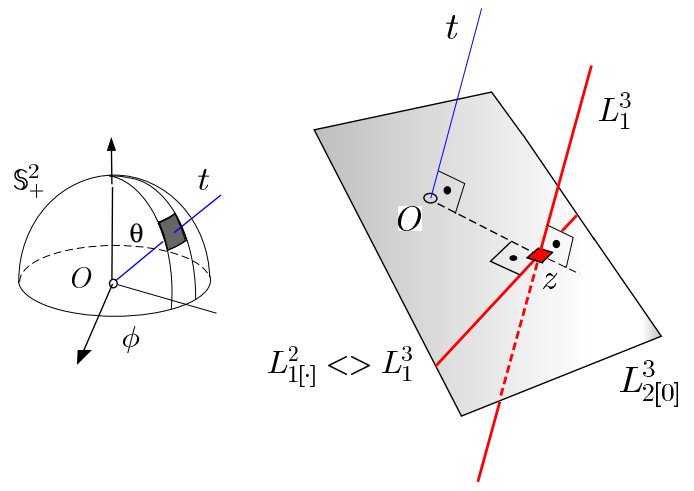

Fig. 1. A point sampled test line $L_{1[\cdot]}^{2}$ in an isotropic pivotal plane $L_{2[0]}^{3}$ through a fixed pivotal point $O$ is equivalent to the classical invariant test line $L_{1}^{3}$ in three dimensions.
As described in Cruz-Orive (2005), point sampled test lines (not to be confused with point sampled intercepts, see for instance Howard and Reed, 2005) have multiple applications, notably to estimate individual particle surface area and volume simultaneously. It seems therefore opportune to explore the relative precision of the pivotal estimators against their current competitors, namely the surfactor (Jensen and Gundersen, 1989) and the nucleator (Gundersen, 1988): this is the purpose - and the new contribution - of the present paper. For the sake of a tractable but still orientating treatment, we adopt a simple geometric particle model. The subsequent results are not intended to be directly applicable to real objects; they rather constitute a tentative analysis of the relative efficiency of the pivotal estimators of surface area and volume.

\section{BACKGROUND AND MODEL}

\section{PIVOTAL ESTIMATORS}

Here we summarize some results from Cruz-Orive (2005). Consider a particle $Y$, namely a compact and connected subset of $\mathbb{R}^{3}$ with piecewise smooth boundary, with (finite) surface area $S(\partial Y)$ and positive volume $V(Y)$. Embed $Y$ in a ball $B_{3}$ of equatorial area $a$ centred at a pivotal point $O$. Take a pivotal plane $L_{2[0]}^{3}$ through $O$ with isotropic normal direction $t$, and generate a uniform random (UR) point $z$ within the equatorial intersection circle $B_{2, t}=B_{3} \cap L_{2[0]}^{3}$. 


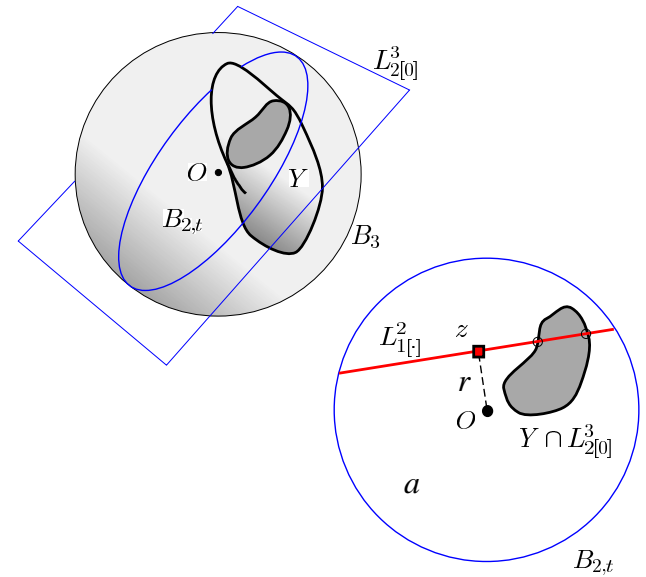

Fig. 2. Theoretical construction of a point sampled test line hitting a particle.

Let $(r, \alpha)$ denote the polar coordinates of $z$ with respect to $O$ in the pivotal plane. Finally, draw a point sampled test line $L_{1[\cdot]}^{2}$ through $z$ and normal to the axis $\mathrm{Oz}$ (see Fig. 2). The joint probability element of the triplet $(r, \alpha, t)$ is

$$
\mathbb{P}(\mathrm{d} r, \mathrm{~d} \alpha, \mathrm{d} t)=\frac{r \mathrm{~d} r \mathrm{~d} \alpha}{a} \cdot \frac{\mathrm{d} t}{2 \pi},
$$

because $z$ is UR on $B_{2, t}$ and independent from the direction $t$, which is UR on the unit hemisphere. The test line $L_{1[\cdot]}^{2}$ is effectively isotropic uniform random (IUR) hitting $B_{3}$, and therefore, if $L_{1[\cdot]}^{2}$ hits $Y \subset B_{3}$, it is also IUR hitting $Y$ (Miles and Davy, 1976). Define the random observations $I:=$ number of intersections between $L_{1[\cdot]}^{2}$ and $\partial Y$, and $I:=$ intercept length determined in $Y$ by $L_{1[\cdot]}^{2}$. Resorting to the pertinent Crofton formulae it follows that

$$
\hat{S}(\partial Y)=2 a I, \quad \hat{V}(Y)=a L,
$$

are unbiased estimators of the particle surface area and volume, respectively.

The preceding construction will be useful in the sequel to compute the variances of the estimators (Eq. 1) for a simple model. In practice, however, we may lay a system of test points with a fundamental tile of area $a$ uniformly at random with an arbitrary orientation on the pivotal plane, and then draw the relevant point sampled test lines (Fig. 3). The corresponding unbiased estimators have the same form (Eq. 1).

A special case of interest is that of a convex particle $Y$ with an interior pivotal point or "nucleolus" $O$. Upon the isotropic pivotal section $Y \cap L_{2[0]}^{3}$, which is almost surely convex, construct the corresponding support set $H_{Y \cap L_{2[0]}^{3}}$, namely the set of points enclosed by the graph of the support function $h(w)$ of the section. Then it holds that

$$
S(\partial Y)=4 \mathbb{E}\left\{\operatorname{area}\left(H_{Y \cap L_{2[0]}^{3}}\right)\right\},
$$

the expectation being over the isotropic orientation distribution of the pivotal plane.

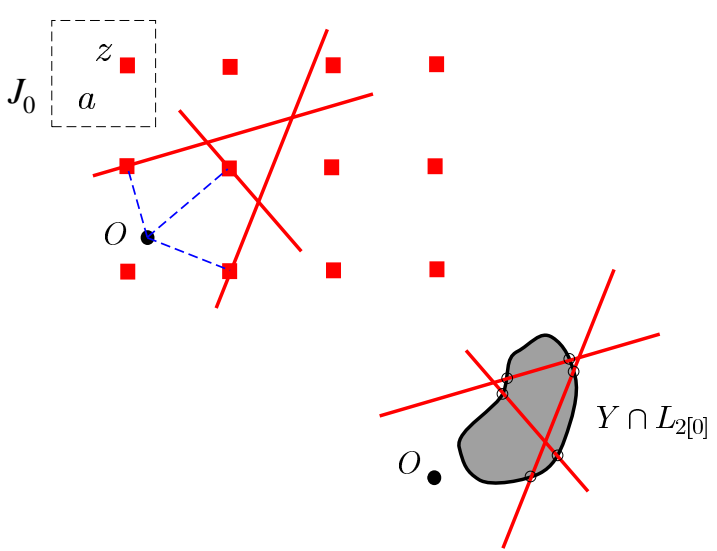

Fig. 3. Point sampled test lines upon a test system on an isotropic pivotal plane, with pivotal point at $O$. The point $z$ is $U R$ within the fundamental tile $J_{0}$ of area a. By Eq. 1 the surface area of the particle would be estimated by $12 a$, and its volume by the total intercept length times $a$.

\section{A SIMPLE MODEL FOR VARIANCE COMPARISONS}

Our particle model is the unit ball $Y$ with an eccentric nucleolus $O$ at a fixed distance $k \in[0,1]$ from the centre of the ball (see Fig. 4). Take an isotropic pivotal plane $L_{2[0]}^{3}$ through $O$. The corresponding pivotal section is a circle which depends only on $k$ and on the colatitude $\theta$ of the normal to the pivotal plane. Isotropy means that the probability element of $\theta$ is

$$
\mathbb{P}(\mathrm{d} \theta)=\sin \theta \mathrm{d} \theta, \quad 0 \leq \theta \leq \pi / 2 .
$$

The radius $r(\theta ; k)$ of the section, and the distance $l(\theta ; k)$ from the section centre $O^{\prime}$ to the pivotal point $O$ have the following expressions,

$$
\begin{aligned}
r(\theta ; k) & =\sqrt{1-k^{2} \cos ^{2} \theta}, \\
l(\theta ; k) & =k \sin \theta
\end{aligned}
$$

respectively. 


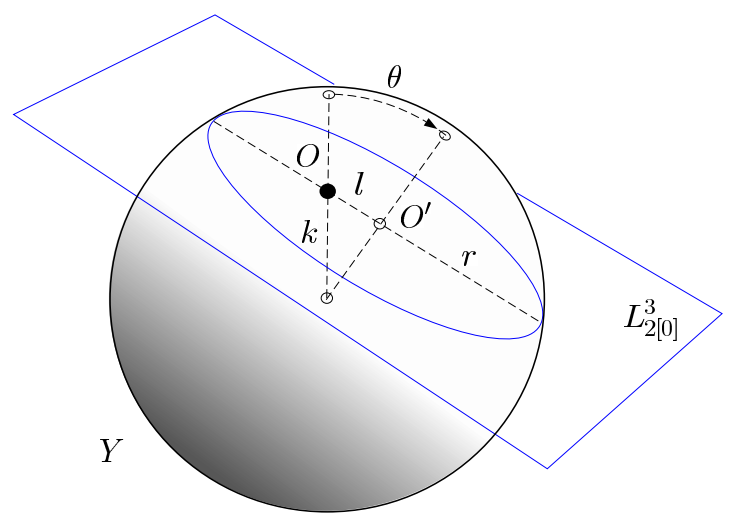

Fig. 4. Spherical particle model with an eccentric nucleolus fixed at $O$ which is adopted as the pivotal point. The subsequent estimators are all based on an isotropic pivotal plane section.

\section{SURFACE AREA ESTIMATORS}

\section{THE PIVOTAL ESTIMATOR OF SURFACE AREA}

Let $h(w, \theta ; k)$ denote the support function of the pivotal section circle $\partial Y \cap L_{2[0]}^{3}$, namely the cardioid,

$$
h(w, \theta ; k)=r(\theta ; k)-l(\theta ; k) \cos w, \quad 0 \leq w \leq 2 \pi
$$

(see Fig. 5). Bearing in mind that

$$
\begin{aligned}
\operatorname{area}\left(H_{\left.Y \cap L_{2[0]}^{3} ; \theta, k\right)}:\right. & =A(\theta ; k) \\
& =\frac{1}{2} \int_{0}^{2 \pi} h^{2}(w, \theta ; k) \mathrm{d} w,
\end{aligned}
$$

and in order to make a fair comparison with the surfactor, instead of basing our estimator directly on Eq. 2, we consider the following unbiased estimator of $S(\partial Y)$ :

$$
\hat{S}_{\text {piv }}(w, \theta ; k)=4 \pi \cdot h^{2}(w, \theta ; k) .
$$

The joint probability element of the pair $(w, \theta)$ is

$$
\begin{aligned}
& \mathbb{P}(\mathrm{d} w, \mathrm{~d} \theta)=\frac{\mathrm{d} w}{2 \pi} \cdot \sin \theta \mathrm{d} \theta \\
& 0 \leq w \leq 2 \pi, 0 \leq \theta \leq \pi / 2,
\end{aligned}
$$

whereby it is readily verified that

$$
C V^{2}\left(\hat{S}_{\mathrm{piv}}(w, \theta ; k)\right)=\frac{4}{3} \cdot k^{2}, \quad k \in[0,1] .
$$

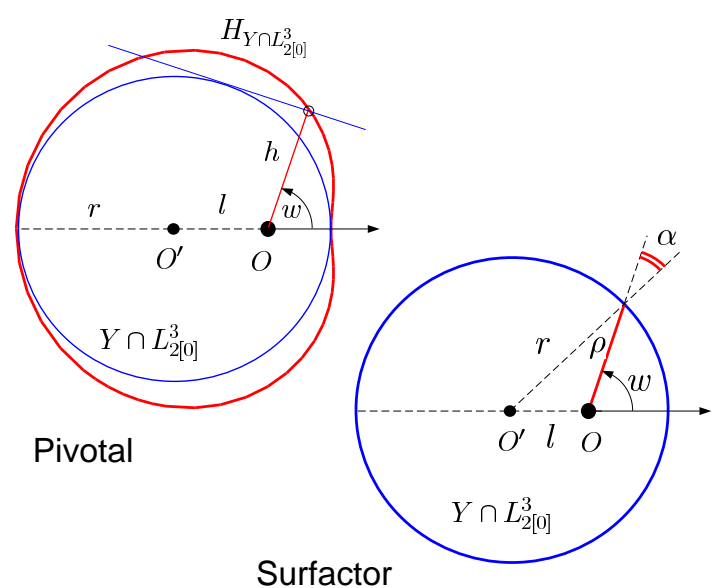

Fig. 5. Pivotal section circle of the particle model shown in Fig.4, with the relevant parameters involved in the pivotal and in the surfactor estimators of surface area, respectively.

\section{THE SURFACTOR}

With reference to Fig. 5, the surfactor estimator of $S(\partial Y)$, (Jensen and Gundersen, 1989; see also Karlsson and Cruz-Orive, 1997), reads,

$$
\begin{aligned}
\hat{S}_{\text {sur }}(w, \theta ; k)= & 4 \pi \cdot \rho^{2}(w, \theta ; k) \\
& \times(1+\alpha(w, \theta ; k) \tan \alpha(w, \theta ; k)),
\end{aligned}
$$

where

$$
\begin{aligned}
& \rho(w, \theta ; k)=r(\theta ; k)[\left(1-\lambda^{2}(\theta ; k) \sin ^{2} w\right)^{1 / 2} \\
&-\lambda(\theta ; k) \cos w], \\
& \alpha(w, \theta ; k)= \sin ^{-1}(\lambda(\theta ; k) \cdot|\sin w|) \in[0, \pi / 2], \\
& \lambda(\theta ; k)=l(\theta ; k) / r(\theta ; k) \in[0,1],
\end{aligned}
$$

and the joint probability element of the pair $(w, \theta)$ is given by Eq. 5, whereby,

$$
\begin{array}{r}
C V^{2}\left(\hat{S}_{\mathrm{sur}}(w, \theta ; k)\right)=\frac{4}{3} \cdot k^{2}+\frac{1}{15} \cdot k^{4}+c(k), \\
k \in[0,1],
\end{array}
$$

where

$$
\begin{aligned}
c(k)=\int_{0}^{\pi / 2} & r^{4}(\theta ; k) \sin \theta \mathrm{d} \theta \int_{0}^{2 \pi} \rho^{4}(w, \theta ; k) \\
& \times \alpha^{2}(w, \theta ; k) \tan ^{2} \alpha(w, \theta ; k) \frac{\mathrm{d} w}{2 \pi} \geq 0,
\end{aligned}
$$

and therefore,

$$
\begin{aligned}
C V^{2}\left(\hat{S}_{\text {sur }}(w, \theta ; k)\right) & \geq \frac{4}{3} \cdot k^{2}+\frac{1}{15} \cdot k^{4} \\
& \geq C V^{2}\left(\hat{S}_{\text {piv }}(w, \theta ; k)\right),
\end{aligned}
$$

see Fig. 7, in which the constant $c(k)$ in the right hand side of Eq. 9 has been evaluated numerically. 
Remark. It is possible to consider more precise versions of the estimators (Eq. 4) and (Eq. 7) by averaging out with respect to the marginal probability element $\mathbb{P}(\mathrm{d} w)=\mathrm{d} w /(2 \pi)$ for each $\theta$. For the pivotal estimator we obtain 4 times the area of the support set $H_{Y \cap L_{2[0]}^{3}}$, namely,

$$
\begin{aligned}
\hat{S}_{\text {piv }}(\theta ; k) & =4 A(\theta ; k) \\
& =4 \pi\left(1-k^{2}+\frac{3}{2} k^{2} \sin ^{2} \theta\right),
\end{aligned}
$$

as expected by virtue of Eqs. 2 and 3. Less obvious, however, is the fact that the corresponding expectation of the surfactor estimator (Eq. 7) with respect to $\mathbb{P}(\mathrm{d} w)$ yields the same result (Eq. 10).

\section{VOLUME ESTIMATORS}

\section{THE PIVOTAL ESTIMATOR OF VOLUME}

We adopt the second estimator in Eq. 1 with $a=S(\partial Y) / 4$. This choice implies that only positive intercept lengths are recorded, namely $L>0$. Equivalently, the sampling point $z$ is chosen uniformly at random in the interior of the support set $H_{Y \cap L_{2[0]}^{3}}$ of the pivotal section, see Fig. 6. Thus,

$$
\hat{V}_{\text {piv }}(\rho, w, \theta ; k)=\frac{1}{4} S(\partial Y) \cdot L(\rho, w, \theta ; k),
$$

is an unbiased estimator of $V(Y)$, where

$$
\begin{aligned}
& L(\rho, w, \theta ; k) \\
& \quad=\left[r^{2}(\theta ; k)-(\rho+l(\theta ; k) \cos w)^{2}\right]^{1 / 2} .
\end{aligned}
$$

On the other hand, the joint probability element of $(\rho, w, \theta)$ is,

$$
\begin{aligned}
& \mathbb{P}(\mathrm{d} \rho, \mathrm{d} w, \mathrm{~d} \theta \mid L>0)=\frac{\rho \mathrm{d} \rho \mathrm{d} w}{S(\partial Y) / 4} \cdot \sin \theta \mathrm{d} \theta, \\
& \rho \in[0, h(w, \theta ; k)), \theta \in[0, \pi / 2], w \in[0,2 \pi) .
\end{aligned}
$$

Note that the unbiasedness of the estimator (Eq. 11) is a consequence of the well known stereological relation $\mathbb{E} L=4 \mathrm{~V} / \mathrm{S}$, (Hostinský, 1925). The estimator (Eq. 11) is of course not practical because, for the ball particle model considered here, if the factor $S(\partial Y)$ in the right hand side of Eq. 11 is known, then $V(Y)$ is also known. The estimator is nonetheless useful for the intended purposes.

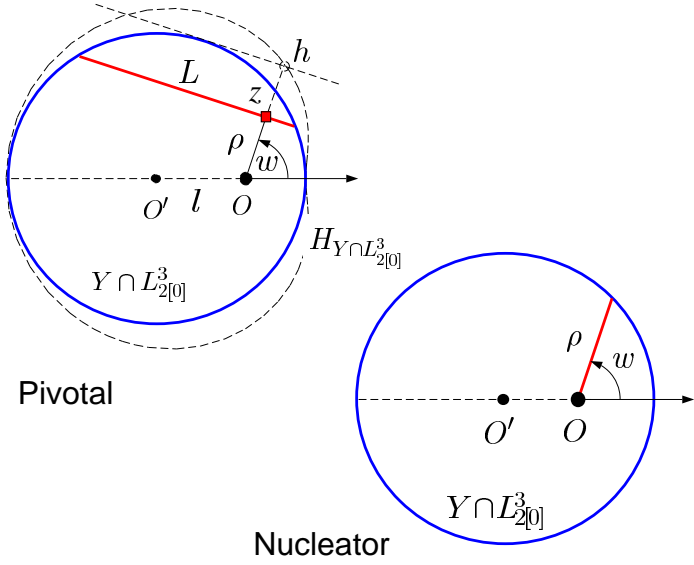

Fig. 6. Pivotal section circle of the particle model shown in Fig. 4, with the relevant parameters involved in the pivotal and in the nucleator estimators of particle volume, respectively.

With the aid of Mathematica ${ }^{\circledR}$, a direct evaluation from Eqs. 11-13 yields,

$$
C V^{2}\left(\hat{V}_{\text {piv }}(\rho, w, \theta ; k)\right)=\frac{1}{8},
$$

independently of the distance $k$ of the pivotal point from the particle centre. The preceding result could have been anticipated bearing in mind that the estimator (Eq. 11) is proportional to the length of the intercept determined in $Y$ by an IUR test line hitting it. This is because a point sampled test line on the pivotal plane is effectively an invariant test line in $\mathbb{R}^{3}$ for any location of the pivotal point, as stressed in the Introduction. The probability element of an IUR chord length $L$ of the unit ball is $\mathbb{P}(\mathrm{d} L)=L \mathrm{~d} L / 2$, $0 \leq L \leq 2$, whereby $C V^{2}(L)=1 / 8$. The argument is general: $C V^{2}\left(\hat{V}_{\text {piv }}(\rho, w, \theta ; k)\right)$ is independent of the position of the pivotal point for any arbitrary particle $Y$; it only depends on the first two moments of the IUR intercept length of $Y$. If $Y$ is not convex, then the relevant intercept may consist of several separate segments, in which case $L(\rho, w, \theta ; k)$ is the sum of the corresponding lengths.

Remark. It is instructive to realise that

$$
\begin{array}{ll}
\mathbb{P}(\mathrm{d} \rho, \mathrm{d} w \mid \theta, L>0) & =\frac{\rho \mathrm{d} \rho \mathrm{d} w}{A(\theta ; k)}, \\
\mathbb{P}(\mathrm{d} \theta \mid L>0) & =\frac{A(\theta ; k) \sin \theta \mathrm{d} \theta}{S(\partial Y) / 4},
\end{array}
$$

and the product of the preceding two conditional probability elements yields the joint one in Eq. 13. Here $A(\theta ; k)$ is defined as in Eq. 3. 

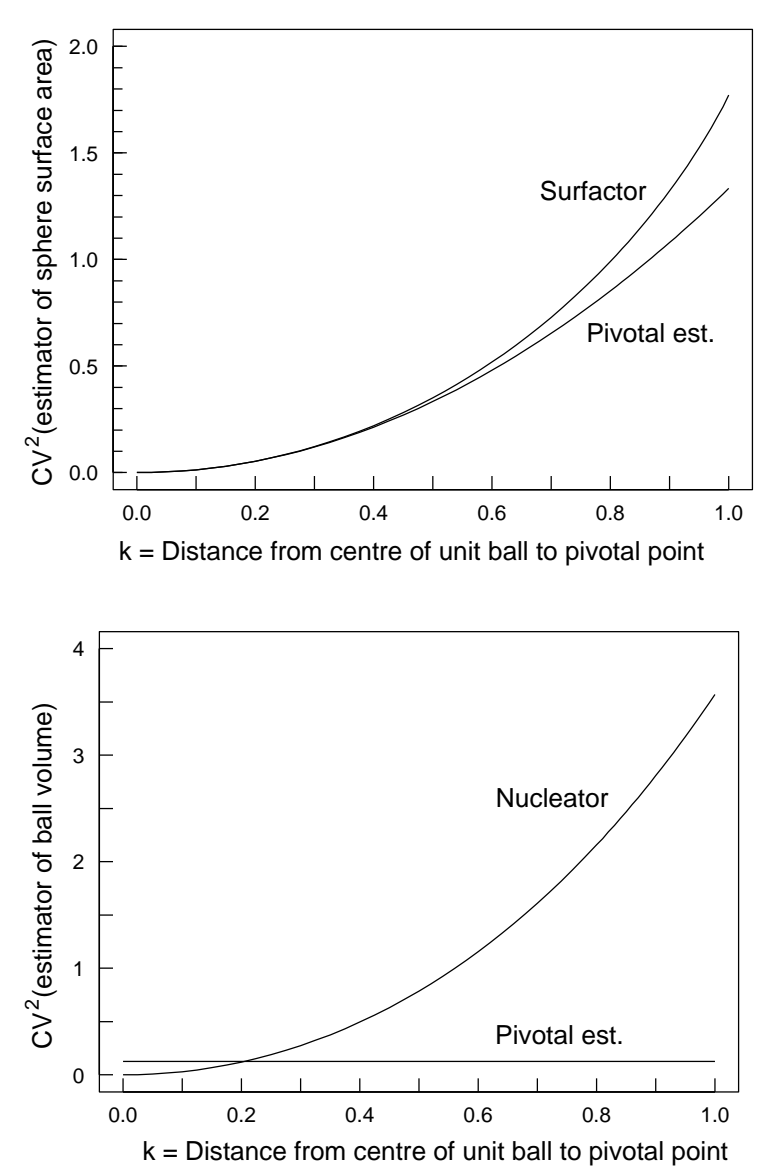

Fig. 7. Upper panel: Graphs of the right hand sides of Eqs. 6 and 9, respectively, showing that the pivotal estimator of surface area is more efficient than the surfactor for the particle model described above (Fig. 4). Lower panel: Graphs of the right hand sides of Eqs. 14 and 16, respectively, showing that the pivotal estimator of volume is more efficient than the nucleator when $k>0.203 \cdots$.

\section{THE NUCLEATOR}

With reference to Fig. 6 we consider the "one-ray" nucleator estimator

$$
\hat{V}_{\mathrm{nuc}}(w, \theta ; k)=\frac{4 \pi}{3} \cdot \rho^{3}(w, \theta ; k),
$$

which is unbiased for $V(Y)$, (Gundersen, 1988). The expression for $\rho(w, \theta ; k)$ is given in Eq. 8. The relevant probability element is given by Eq. 5 , whereby we obtain

$$
\begin{array}{r}
C V^{2}\left(\hat{V}_{\text {nuc }}(w, \theta ; k)\right)=3 k^{2}+\frac{3}{5} k^{4}-\frac{1}{35} k^{6}, \\
k \in[0,1],
\end{array}
$$

see Fig. 7.

In practice it is much more efficient to consider two rays $\rho(w, \theta ; k)$ and $\rho(w+\pi, \theta ; k)$, but in Eq. 15 we have chosen one because then the efficiency comparison with the pivotal estimator (Eq. 11) looks more fair. For the two ray nucleator we obtain

$$
C V^{2}\left(\hat{V}_{\mathrm{nuc}}(w, \theta ; k)\right)=\frac{9}{5} k^{4}-\frac{18}{35} k^{6}, \quad k \in[0,1],
$$

for the ball particle model considered here.

\section{DISCUSSION}

At least for the unit ball particle model considered here with a nucleolus at a distance $k$ from the centre: (i) The one-ray pivotal estimator (Eq. 4) of surface area is simpler and more efficient than the surfactor. (ii) The one-intercept pivotal estimator (Eq. 11) of volume is more efficient than the one-ray nucleator for $k>0.203 \cdots$. Even for the two-ray nucleator (Eq. 17) the pivotal estimator is more efficient for $k>0.542 \cdots$.

If $Y$ is a convex body, then for the first estimator (Eq. 1) it is easy to show that

$$
C V^{2}(\hat{S}(\partial Y))=\frac{a}{S(\partial Y) / 4}-1 .
$$

On the other hand, if $Y$ is the unit ball then for the second estimator (Eq. 1) we have

$$
C V^{2}(\hat{V}(\partial Y))=\frac{9}{8} \cdot \frac{a}{S(\partial Y) / 4}-1
$$

The preceding formulae take into account that the estimators (Eq. 1) may be equal to zero if the test line fails to hit $Y$. If we set $a=S(\partial Y) / 4$, then $\hat{S}(\partial Y)=$ $S(Y)$ because $I=2$ with probability 1 , and trivially $C V^{2}(\hat{S}(\partial Y))=0$. On the other hand $\hat{V}(Y)$ is in this case the right hand side of Eq. 11, and Eq. 19 becomes Eq. 14.

Finally we note that, unlike Eq. 18, Eq. 6 depends on the position of the pivotal point because the estimator (Eq. 4) is not a direct function of the intersection between the test line and $Y$.

\section{ACKNOWLEDGMENTS}

This research was supported by the Spanish Ministry of Education and Science I+D Project no. MTM2005-08689-C02-01.

\section{REFERENCES}

Cruz-Orive LM (2005). A new stereological principle for test lines in 3D. J Microsc 219:18-28.

Gundersen HJG (1988). The nucleator. J Microsc 151:3-21.

Hostinský B (1925). Sur les probabilités géométriques. Publ Fac Sci Univ Masaryk 50. Brno. 
Howard CV, Reed MG (2005). Unbiased Stereology. 2nd edn. Abingdon, Oxon: Garland Science.

Jensen EB, Gundersen HJG (1989). Fundamental stereological formulae based on isotropically orientated probes through fixed points with applications to particle analysis. J Microsc 153:249-67.

Karlsson LM, Cruz-Orive LM (1997). Estimation of mean particle size from single sections. J Microsc 186:121-32.

Miles RE, Davy PJ (1976). Precise and general conditions for the validity of a comprehensive set of stereological fundamental formulae. J Microsc 107:211-26.

Varga O (1935). Integralgeometrie 3. Croftons Formeln für den Raum. Math Zeitschrift 40:387-405. 